\title{
Complexité : jalons pour une définition
}

\section{Complexity: landmarks toward a definition}

\author{
Jean-Claude Sallaberry ${ }^{1}$ \\ ${ }^{1}$ Professeur émérite à l'Université de Bordeaux — IMS-CNRS, UMR 5218/ISCC, équipe CIH (cognitique et ingénierie \\ humaine), groupe cognitique (École Nationale Supérieure de Cognitique - 109 avenue Roul - 33400 Talence - France)
}

RÉSUMÉ. Cet article constitue une mise à jour d'un travail sur la complexité qui se précise, se complète peu à peu (1999, 2003 2018b notamment). L'une des idées de base étant que la modélisation du fonctionnement d'une société par le schéma en trois moments (théorie de l'institution), à condition de boucler le troisième moment sur le premier, décrit une dynamique de système autopoiétique (cf. Varela). Dès lors, les boucles autopoiétiques étranges proposées par Varela peuvent être adaptées à la modélisation d'une société, enrichissant la reprise du co-engendrement des éléments et de la forme (Barel et Castoriadis) - caractéristique, d'après ce dernier, de la complexité.

Traditionnellement, en Sciences physiques, on procède à la caractérisation d'une grandeur avant d'énoncer sa définition (on affine les bords de la notion avant de la porter au concept). Nous commencerons donc par rappeler le travail de caractérisation opéré par Le Moigne et par Morin. Nous rappellerons ensuite les propositions de Nicolis et Prigogine, puis de Castoriadis. Nous poursuivrons avec l'intervention des boucles autopoiétiques étranges. Pour la discussions, nous aborderons enfin la question du lien invariant-concept (Vergnaud) au sujet de la complexité.

ABSTRACT. This article constitutes an updating of a work about complexity, which becomes more precise and little by little more complete $(1999,2003,2018 \mathrm{~b}$ in particular). One of the basic ideas is that modelisation of society functioning is a three-stage (three "moments") scheme (institutional theory), on condition that the third stage (moment) comes back on the first one in a loop, it describes the dynamic movement of an autopoietic system (cf. Varela). Thus, the strange autopoietic loops proposed by Varela can be adapted to the modelisation of society, enriching the idea of cogenerating elements and shape (Barel and Castoriadis), which is characteristic of complexity, according to Castoriadis.

Traditionally in Physics, we characterize a size before stating its definition (making the sides of a notion more precise before it becomes a concept). We will begin with mentioning Le Moigne and Morin's work on characterization. Then, we will study Nicolis and Prigogine's propositions, and finally Castoriadis's work. We will continue with the coming up of strange autopoietic loops. For the discussion, we will finally come to the matter of the link drawn between unvarying element and concept (Vergnaud) applied to complexity.

MOTS-CLÉS. Complexité, boucles autopoiétiques étranges, institution, structure, système.

KEYWORDS. Complexity, strange autopoietic loops, institution, structure, system.

\section{Introduction}

On pourrait rappeler la définition du CNRLT pour l'adjectif complexe : "Composé d'éléments qui entretiennent des rapports nombreux, diversifiés, difficiles à saisir par l'esprit, et présentant souvent des aspects différents. $»^{2}$ Comme toute définition du genre dictionnaire (formulation nécessairement brève), elle a du mal à caractériser avec précision la notion ciblée. En reprenant la tradition de la Physique, nous appuierons une approche de définition sur une caractérisation préalable.

Ainsi, le début sera consacré à une caractérisation de la complexité, prenant appui sur Le Moigne [LEM 90] et Morin [MOR 90]. Puis, deux propositions de définition, celles de Nicolis-Prigogine et Stengers [PRI 79] et celle de Castioriadis [CAS 93]), seront exposées. Une nouvelle approche, à partir des boucles autopoiétiques étranges, sera ensuite proposée. La convergence entre cette approche et celle de Castoriadis permettra alors l'esquisse d'une définition (pour un système complexe). La discussion s'appuiera sur la question du lien invariant-concept. 


\section{Caractérisation de la complexité}

Le Moigne et Morin effectuent un important travail de caractérisation. Les points de départ sont différents, mais les perspectives qu'ils tracent convergent. Ils cosignent d'ailleurs l'ouvrage L'intelligence de la complexité [LEM 99].

\subsection{Le Moigne}

Dans son ouvrage de 1990, La modélisation des systèmes complexes [LEM 90], Le Moigne caractérise la complexité à partir de l'opposition compliqué/complexe :

- on peut simplifier un système compliqué, pour découvrir son intelligibilité (processus d'explication) ;

- on doit modéliser un système complexe pour construire son intelligibilité (processus de compréhension), ainsi que celle des deux processus décomposition/composition (ou disjonction/conjonction) ${ }^{3}$ :

- le compliqué est décomposable, par décomposition ou disjonction, on obtient un « décomposé » simple ;

- le complexe est indécomposable, par composition ou conjonction ;

- on peut éventuellement obtenir un «implexe » (qui est un «indécomposé »).

En outre, cette idée de modélisation est essentielle. Dans la physique classique, le travail du scientifique était déjà, bien sûr, un travail de modélisation : il fallait par exemple raisonner aux limites (en excluant par exemple les frottements, pour concevoir le principe d'inertie). Mais on avait l'impression qu'à partir de l'observation on pouvait caractériser, outiller un phénomène. Bachelard ([BAC 34] [BAC 38]) a bien sûr souligné que l'expérience n'était pas première — qu'il fallait toute une construction théorique, nantie d'hypothèses, pour concevoir une expérience susceptible d'infirmer ou de vérifier une hypothèse ${ }^{4}$. Mais la possibilité d'observation du phénomène pouvait laisser croire que la science le décrivait, en quelque sorte. Avec la physique des particules, on ne peut plus observer que des effets de l'événement réel (les clichés de chambre à bulle, par exemple, permettent d'échafauder des hypothèses sur ce qu'a pu produire un choc de particules). Il est dès lors clair qu'on ne peut que modéliser. Il en est de même pour tout ce qui est complexe . $^{5}$

Le Moigne, à partir de son important travail sur la théorie des systèmes, nous apporte ainsi l'important distinguo compliqué/complexe, ainsi que l'idée de modélisation.

\subsection{Morin}

Morin [MOR 90] mène lui aussi un travail de caractérisation important, en posant notamment ( $\mathrm{p}$. 98-100) les «trois principes », que l'on peut considérer comme des critères caractérisant la complexité :

- principe dialogique (qui permet d'associer deux termes à la fois complémentaires et antagonistes),

- principe de récursion organisationnelle (un processus récursif est un processus où les produits et les effets sont en même temps causes et producteurs de ce qui les produit),

3 II mène également un important travail au niveau épistémologique, en fondant clairement une épistémologie constructiviste, englobant une épistémologie positiviste (1995) [LEM 95].

${ }^{4}$ Voir par exemple [BAC 38] p.14 : « C'est précisément ce sens du problème qui donne la marque du véritable esprit scientifique. Pour un esprit scientifique, toute connaissance est une réponse à une question. S'il n'y a pas eu de question, il ne peut y avoir de connaissance scientifique. Rien ne va de soi. Rien n'est donné. Tout est construit. » 
- principe hologrammatique (par exemple, chaque cellule d'un organisme biologique contient la totalité de l'information génétique de cet organisme).

On pourrait ajouter, à ces trois principes, celui de non-séparabilité des variables ${ }^{6}$ Mais le plus important est le parallèle que l'on peut établir avec les trois critères par lesquels dès 1968 Piaget caractérise la notion de structure [PIA 68]. Car dès lors, la structure devient une notion fondamentale et interdisciplinaire, un outil de modélisation disponible pour la pensée moderne. Ce n'est plus seulement, selon les cas, une succession d'invariants dans un conte comme le propose Propp [PRO 28]), la langue considérée comme un système par Saussure [SAU 15]), un schème unique «derrière le chaos des règles et des coutumes » pour Lévi-Strauss [LEV 58]), les opérations qui structurent un ensemble dans la pensée des Bourbaki [BOU 70], un organisme vivant tel que le conçoit Piaget [PIA 68].), c'est "quelque chose" qui se caractérise par les critères :

- de totalité (le quelque chose est "plus“ que la simple juxtaposition de ses éléments)

- de transformation (le quelque chose est habité par des transformations et se transforme)

- d'autorèglage (malgré les transformations, il y a quelque chose qui se conserve, au niveau du fonctionnement)

Autrement dit, la notion de structure, nantie des critères de Piaget, non seulement se généralise, mais encore affine ses bords et se rapproche d'une définition possible — donc d'un concept.

On peut considérer qu'à partir des trois critères, l'idée que l'invariant peut résider dans le fonctionnement a été décisive dans l'essor, au XXe siècle, du structuralisme puis de la théorie des systèmes, mais aussi de la biologie et des sciences humaines et sociétales [SAL 18b].

De même, les trois principes de Morin jouent (ou peuvent jouer) le rôle de critères pour le complexe (ou la complexité). Nous renvoyons bien sûr à l'impressionnant travail de ces deux auteurs, notre propos n'étant pas d'en faire l'économie. On peut considérer que tous deux mènent ainsi le travail de caractérisation nécessaire à toute tentative d'élaboration d'une définition.

\subsection{L'apport d'Atlan}

Atlan parle surtout d'auto-organisation. Dans une première tentative de caractérisation, en 1979 [ATL 79], il note : «Si, sous l'effet de... perturbations aléatoires, le système, au lieu d'être détruit ou désorganisé, réagit par un accroissement de complexité et continue de fonctionner, nous disons alors que le système est auto-organisateur. » (p.167). On retiendra de ce travail l'appui à la notion de système, ainsi que le lien à celle de complexité. Malgré ce lien, il ne semble pas envisageable d'identifier, a priori, auto-organisation et complexité.

\section{Deux conceptions/définitions de la complexité}

En Physique, la méthode habituelle consiste, une fois qu'elle est caractérisée, à définir une grandeur. En ce qui concerne la complexité, une première tentative de conception/définition est due à Nicolis et Prigogine [NIC 92], une seconde à Castoriadis [CAS 93] (à partir des travaux de Barel [BAR 75] et Castoriadis [CAS 93]). Conception/définition, parce qu'à partir d'une conception suffisamment précise, il n'y a qu'un pas pour tenter une définition (il suffit d'une reformulation).

\subsection{Le cas d'un système physique - complexité et imprévisibilité (la complexité comme bifurcation sur la courbe représentative du système)}

La complexité est référée par Nicolis et Prigogine [NIC 92] à des phénomènes d'autoorganisation. Prenons l'exemple comme celui des cellules de Bénard [NIC 92] : il s'agit de cellules

\footnotetext{
${ }^{6}$ Cf. [ESP 84] et [SAL 18b].

7 Pour un travail plus approfondi sur l'apport d'Atlan, voir [SAL 18b] chap. VII. 
provoquées (lors du chauffage d'un fluide limité par deux plaques planes) par des courants de convection ${ }^{8}$. Ils parlent à ce propos de rupture de symétrie (de l'espace) ${ }^{9}$ et font remarquer que si l'apparition des cellules relève d'un déterminisme strict, on ne peut en revanche prévoir le sens de rotation d'une cellule. Ainsi, deux états sont possibles pour un même ensemble de paramètres. En généralisant cette remarque, on pourra lier le caractère imprévisible de certains aspects d'un phénomène à l'existence d'une bifurcation sur la courbe représentative du système considéré dans l'espace des phases ${ }^{10}$.

Une première conception de la complexité se caractérise ainsi par le constat ou l'hypothèse d'une imprévisibilité partielle du comportement d'un système, le critère utilisable étant l'existence (ou non) d'une bifurcation sur la courbe représentative dans l'espace des phases.

Si l'hypothèse d'une imprévisibilité partielle peut tout à fait participer à la caractérisation d'une situation de formation ou d'une autre situation sociétale, le critère de la bifurcation (associé à l'hypothétique possibilité d'une courbe représentative supposant des variables définies et séparées) semble plus difficile à concevoir. Par ailleurs, la suggestion de Castoriadis [CAS 93] permet un enrichissement des perspectives, tant au niveau théorique qu'au niveau épistémologique.

\subsection{Le cas d'un système sociétal}

Pour aborder la suggestion de Castoriadis [CAS 93], il faut rappeler l'articulation des niveaux logiques individuel et collectif ainsi que quelques éléments de la théorie de l'institution.

Si l'on reprend la proposition du cercle de Vienne ( $c f$. Russel et Whitehead [WHI 10]), en considérant qu'on ne peut penser de la même façon un élément et sa classe, on en vient à remarquer que l'on ne peut penser non plus de la même façon l'individu et la société. Le niveau logique individuel est celui de 'l'atome social' - puisque le mot 'individu' contient l'hypothèse que l'on ne peut le diviser. Le niveau logique collectif concerne les groupes, les organisations, la société et les «phénomènes collectifs » en général.

La théorie de l'institution fournit un outillage précieux et original pour cette prise en compte des deux niveaux logiques dans toute tentative de modélisation du fonctionnement sociétal.

\subsubsection{Fonctionnement d'une règle (modélisation institutionnelle de l'articulation individuel-collectif)}

Dans bien des groupes «post-soixante-huit » régnait une certaine frénésie de prise de décision. On décidait beaucoup, mais le plus souvent, quand chaque membre se retrouvait, seul, à devoir appliquer la décision, il «statuait» pour son propre compte et décidait, du point de vue de sa vie personnelle, de suivre cette décision, ou non. Cette anecdote peut étonner. Une décision collective qui n'engage pas ceux qui la prennent est-elle une vraie décision? Cet engagement, cette égalité devant la décision prise caractérisent le premier moment du fonctionnement d'une règle — ou d'une décision devenue règle, le moment de l'Universel. Cela s'inscrit sans doute dans une culture démocratique. En revanche, l'hésitation à appliquer correspond au second moment, celui du Particulier. Le sujet se retrouve face à la règle et va l'appliquer, à sa façon... Si le moment de l'Universel (ou de $1^{\prime}$ Universalité) est celui où une règle s'énonce en tant que la même pour tous, le moment du Particulier (ou de la Particularité) correspond à la situation du sujet qui doit appliquer la règle. L'exemple montre bien la complémentarité des deux moments.

Ce fonctionnement peut se représenter par le schéma en trois moments représenté à la figure $\mathrm{n}^{\circ} 1$ :

\footnotetext{
${ }^{8}$ Atlan cite le « rôle pionnier de Turing et des tourbillons de Bénard » (p. 14) [ATL 11].

9 L'exemple de la réaction « BZ » renvoie à une rupture de symétrie vis-à-vis du temps [VID 92].

10 Espace comportant autant de dimensions que le système admet de variables descriptives. 


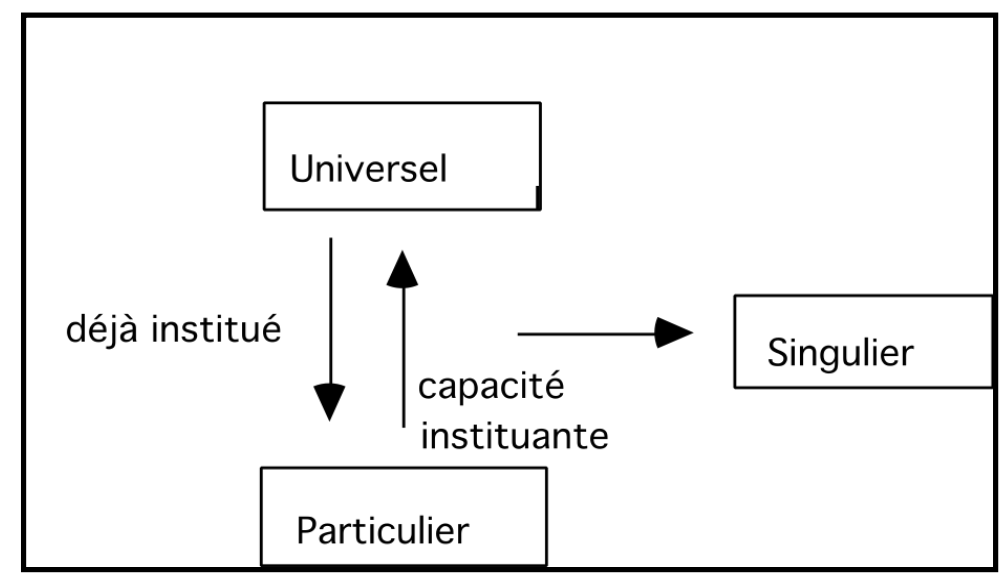

Figure 1. Schéma en trois moments

Les deux flèches verticales indiquent l'interaction entre le premier et le deuxième moment. La flèche horizontale indique la production du troisième moment.

Il est clair que les deux premiers moments interagissent : la façon qu'une règle a d'être appliquée modifie la règle, ou tout au moins contient (potentiellement) la possibilité de la modifier. Cette interaction produit le troisième moment, celui du Singulier (ou de la Singularité).

Outre la symbolisation de l'interaction entre le premier et le deuxième moment, les deux flèches verticales peuvent être interprétées de façon plus précise. La flèche descendante représente le déjà institué (partout où l'être humain arrive, il y a déjà des règles, des normes... il ne choisit ni sa famille, ni la société dans lesquelles il advient. La flèche ascendante illustre la capacité instituante (chacun peut interpréter la règle, proposer des modifications, de nouvelles règles...) ${ }^{11}$. La flèche horizontale indique la production du troisième moment, de façon analogue à la flèche de la réaction chimique (deux réactifs produisent deux produits) ${ }^{12}$. En effet, l'interaction entre le premier et le deuxième moment produit le troisième moment, celui du Singulier (ou de la Singularité). C'est le moment de l'émergence des formes collectives : nouvelle formulation de la règle, nouvelle façon de la vivre et de l'appliquer, nouvelle représentation collective. Bien entendu, le caractère nouveau n'a rien d'automatique. Le fonctionnement en trois moments peut fort bien reproduire du même. La nouveauté ressemble alors au déjà-là comme deux gouttes d'eau. C'est le changement 1 , ou faux changement, repéré par la théorie des systèmes.

Ce type de fonctionnement est aussi celui d'une norme - on pourrait caractériser, sinon définir, une norme comme étant une règle non écrite (voire non formulée). La façon de s'habiller, la façon de se comporter dans certaines situations, sont régies, suivant la société et le milieu sociétal, par des normes - qui peuvent être plus insidieuses que des règles, puisque non formulées clairement.

\subsubsection{Application à l'institution, à la langue}

Le terme institution, tel que Lourau le définit [LOU 70], peut prendre plusieurs sens, dont le plus courant est celui d'organisation. Quand on entend parler de l'institution Saint Joseph, on comprend qu'il s'agit d'une organisation, qui se trouve être probablement un établissement scolaire privé, catholique sans doute. Pour la suite, convenons qu'institution désigne non l'organisation elle-même, mais plutôt sa façon de fonctionner : les règles et les normes qui lui permettent de subsister, de ne

\footnotetext{
11 L'idée de l'interaction instituant-institué est due à Castoriadis (cf. Cardan [CAR 65]) ; elle est reprise par Lourau [LOU 70]. Sallaberry propose de modéliser ce fonctionnement par le schéma en trois moments [SAL 96] [SAL 03].

12 En poursuivant cette illustration, on pourrait considérer qu'à l'instar d'une réaction chimique réversible - qui se stabilise dans un équilibre - le troisième moment est dans la situation de l'équilibre. La flèche horizontale indiquerait alors la production de cet équilibre. Mais comme le précise le $\S 2.3$, il ne faut pas considérer le troisième moment comme " définitif »; ce qui s'est stabilisé provisoirement entre en interaction avec le moment de la Particularité.
} 
pas exploser ou imploser... Une définition générale possible serait dès lors celle « d'ensemble de normes, qui tend à se reproduire en tant que dominant». Les règles sont souvent énoncées, voire écrites, les normes sont plus implicites ; dans les deux cas, ces systèmes (de règles et de normes) sont présents dans les têtes des membres de l'organisation - Castoriadis [CAS 75] le rappelle avec son fameux titre : L’institution imaginaire de la société.

Le schéma en trois moments (Figure 1) modélise bien entendu le fonctionnement de toute institution, le troisième moment étant celui où émerge une institution éventuellement remaniée, éventuellement nouvelle.

La langue est une institution particulière, puisqu'elle permet de décrire toute organisation, toute institution, tout objet... Elle est le vecteur principal d'une culture, qu'elle représente.

L'application du schéma en trois moments à la langue (schéma II) permet de comprendre que le moment de l'Universalité correspond au niveau de la structure : c'est le moment où la langue se présente comme un lexique (ensemble de mots permis, présentés dans le dictionnaire) et une syntaxe (ensemble de règles permettant d'associer les mots entre eux pour constituer des phrases). Le niveau du moment de la Particularité devient alors celui de l'événement : le sujet, en créant une parole, peut inventer de nouveaux mots, de nouvelles façons de dire les choses... Quant au moment de la Singularité, il est celui d'une langue vivante, puisqu'elle se transforme peu à peu en fonction des usages. Du point de vue niveau, il représente à la fois celui de l'événement (lorsqu'un changement dans la façon de dire telle ou telle chose survient) mais aussi celui de la structure (qui se reproduit, ou si l'on veut être plus précis, il faut rappeler que l'autoréglage va assurer le maintien du fonctionnement, malgré les transformations).

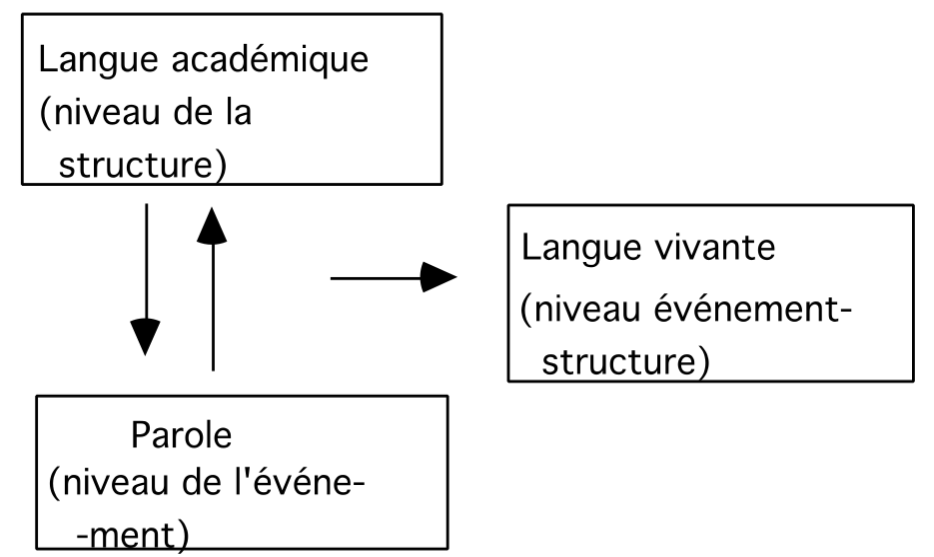

Figure 2. Schéma en trois moments appliqué à la langue - schéma II La signification des flèches est la même que pour le schéma I (Figure 1)

Mais, plus important, l'exemple de la langue montre que sans la structure que constituent lexique et syntaxe, le sujet ne peut créer, inventer une parole originale et irremplaçable. Un sujet humain ne peut parler que dans une langue à la fois. S'il est bilingue, il parle soit dans une langue soit dans l'autre - Aragon disait que c'était alors l'homme de deux destins, ou d'un destin traduit (interview sur France Culture).

En conjuguant alors le premier schéma et le second schéma, il devient clair que la liberté ne peut se concevoir sans l'ensemble de règles et d'institutions qui spécifie une culture, une société. Il devient clair que la pensée n'est possible que dans une culture (ne peut émerger que dans une culture). 


\subsection{Les deux conceptions du troisième moment}

Il est tentant de faire du troisième moment celui de l'institutionnalisation (Hess-Savoye [HES 81], p.56 ${ }^{13}$ ). Une telle interprétation est d'ailleurs dans la « logique » hégélienne (l'opposition entre thèse et antithèse se résout dans la synthèse). Une telle interprétation est en contradiction relative avec celle que nous venons de développer. Non par l'aspect stable, durable, d'un nouvel institué, puisque l'interaction avec la capacité instituante peut fort bien le laisser inchangé. Mais parce que si le troisième moment est celui de l'institutionnalisation, cela signifie que nécessairement le nouvel institué doit durer.

La notion d'émergence d'un état global du système (Varela [VAR 89a]) peut être reprise avec profit pour interpréter le troisième moment, celui de la Singularité ou du Singulier — le moment de l'unité négative, car si le moment du Particulier constitue la négation du premier moment (l'idée même d'un point de vue particulier nie le caractère universel), le moment du Singulier réalise en quelque sorte la synthèse de toutes les particularités. Il faut insister sur cet aspect distributif de la Particularité : il y a sans aucun doute un moment de la Particularité, mais il y a une grande quantité de particuliers (tous ceux qui, un jour ou l'autre, ont maille à partir avec la règle ou avec la norme...). Le troisième moment devient alors celui de l'émergence d'un état global de la règle lorsqu'il s'agit d'une règle. Dans le cas général, il devient de moment de l'émergence d'une forme collective (d'une forme de socialité : nouvelle formulation de la règle, nouvelle façon de la vivre et de l'appliquer, nouvelle représentation collective) ${ }^{14}$.

Cela dit, il n'est pas sûr que la forme qui émerge soit pérenne. On peut penser, au contraire, que non seulement les formes vont devoir être investies, recevoir de l'énergie et des soins pour survivre, mais encore que bien des formes ne seront qu'évanescentes, parce que non repérées par les acteurs qui pourtant les font émerger. C'est ce que l'on peut observer au niveau des groupes, c'est aussi ce que l'on peut observer au niveau des états - tous les changements de régime politique ne "réussissent" pas... Pour qu'une forme dure, il faut qu'elle soit non seulement repérée, mais nommée, investie, décrite, outillée. Il a fallu trois années, de 1789 à 1792, pour que la forme république puisse se construire et s'instituer — et on pourrait considérer avec Furet ([FUR 78], p.15-16) qu'il a fallu un siècle pour qu'elle se mette vraiment en place, dans les difficultés que l'on sait, notamment quant au fait que ce sont les adversaires du changement qui reviennent au pouvoir à la chute de l'Empire.

Ce n'est que lorsqu'elle dure qu'une forme assume le risque de se scléroser et que l'on va pouvoir observer les effets de l'institutionnalisation — avec "l'intervention" de l'effet Mülhmann".

\subsection{Complexité et co-engendrement éléments-forme (co-engendrement ou co-émergence éléments-forme comme critère de la complexité)}

L'idée de co-engendrement des éléments et de la forme naît de la collaboration entre Barel ([BAR 75] [BAR 87] [BAR 89]) et Castoriadis [CAS 93]. L'idée est que la polis « est impossible sans les politai - les citoyens - lesquels, pourtant, ne peuvent être fabriqués que dans et par la polis. » C'est l'exemple de l'Athènes du Ve siècle avant Jésus Christ. Mais le phénomène est similaire pour les nouvelles cités qui émergent en Occident autour de l'an mille. Le bourg libre est inconcevable sans les proto-bourgeois, qui sont inconcevables en dehors du bourg.

Cette idée (cette hypothèse), qui se construit au fil des échanges entre les deux chercheurs ${ }^{16}$, est énoncée et publiée en 1993 par Castoriadis [CAS 93] (dans l'ouvrage collectif dédié à la pensée de Barel), qui en profite pour réfléchir à la complexité.

13

"Quant au troisième moment, celui de la singularité, la négation de la négation, autrement dit c'est l'institutionnalisation. "

${ }^{14}$ Cette émergence d'une forme a un fort caractère imaginaire, souligné par Castoriadis [CAS 75].

15 cf. [SAL 18b], chap.V § II-2-3.

(C) 2021 ISTE OpenScience - Published by ISTE Ltd. London, UK - openscience.fr 
Pour employer une formulation rapide et précise, serait déclaré complexe un système pour lequel on peut observer le co-engendrement des éléments et de la forme. Dans ce cas, l'état global dépend des états locaux, qui dépendent eux-mêmes de l'état global.

Pour enrichir la formulation «rapide et précise » de l'alinéa précédent : Castoriadis [CAS 93], pour caractériser la complexité, après avoir préféré le terme de «création » à celui d' ' émergence », rappelle (p. 59) que lors de la création d'une nouvelle forme, cette " apparition » a un « caractère « intrinsèquement circulaire », d'où l'impossibilité de la «produire » ou de la «déduire » d'éléments déjà donnés - car les «éléments » présupposent la forme, laquelle présuppose les « éléments ». » Il prend comme exemples le «cercle» ADN-protéines et la «création socialhistorique » de la polis. Il souligne (1993, p.70) que l'ouvrage de Barel [BAR 75] « est sous-tendu, et tendu, par les deux questions majeures : qu'est ce qui tient une société ensemble et qu'est-ce qui en fait une société ; et comment et pourquoi y a-t-il émergence du nouveau dans l'histoire ? » Il poursuit en commentant le travail de Barel [BAR 75]: «Le surgissement de la ville médiévale est reconnu comme «discontinuité majeure» (p.74 et p.165 sq), où l'on discerne l' «émergence d'éléments nouveaux » (p.169). Cette discontinuité se tisse avec une «continuité » (1.c. et p.505 et 574), puisqu'elle contient... des éléments hérités... Ces différents éléments n'entretiennent pas entre eux des relations simples : ni du point de vue de la «causalité » puisque, ici, la «causalité » est « circulaire » (p.76) ... et que cette circularité est en même temps une «genèse » ([CAS 93] p.77); ni du point de vue de la signification, puisqu'il y a «indécidabilité » (passim. J'ajouterais: indécidabilité du point de vue ensidique ${ }^{17}$ — et la raison en est le caractère magmatique des significations). Il y a « co-engendrement » (p.304 et 322). » ([CAS 93] p.70 et 71)

Il précise même sa position ([CAS 93] p.61). «J'ai tenté de dire, jusqu'ici, pourquoi certaines des tentatives courantes de préciser le sens de la «complexité » ne me semblent pas satisfaisantes. Je dois maintenant énoncer ce qui me paraît être la raison de cet état de choses. Elle se trouve, je pense, en ceci que les phénomènes (ou objets) considérés comme «complexes" sont tels parce qu'ils relèvent d'une caractéristique plus profonde et plus générale de tout objet, et de l'être en général : leur caractère magmatique. Nous dirons qu'un objet est magmatique lorsqu'il n'est pas exhaustivement et systématiquement ensidisable. »

En résumé, un objet non ensidique (l'humain, le sociétal) apparaît complexe. Or, un tel objet fonctionne par co-engendrement des éléments et de la forme. Il semble donc possible, sur la suggestion de Castoriadis, de formuler l'hypothèse que la complexité d'un « objet » humain et/ou sociétal est caractérisé par un fonctionnement au co-engendrement des éléments et de la forme ${ }^{18}$.

\subsection{Bilan partiel et intervention des deux théories dans l'hypothèse}

En reprenant les deux conceptions/définitions, nous pourrions dire que lorsqu'un système (ou une organisation, ou une situation) peut être caractérisé par une courbe représentative dans l'espace des phases (il est donc «ensidisable» pour Castoriadis), le critère de l'existence (ou non) d'une bifurcation sur la courbe est pertinent pour caractériser la complexité. Dans le cas contraire (impossibilité de concevoir une courbe représentative, et même un espace des phases, vu la vanité

\footnotetext{
16 Barel [BAR 87] [BAR 89] s'interroge sur la relation entre un système et des éléments qui semblent contenir, sinon le système du moins son essence (sa culture), et pose l'hypothèse que les éléments sont, eux aussi, des systèmes.

17 Le qualificatif 'ensidique' est l'abréviation de 'ensembliste-identitaire', qui renvoie en gros à la logique formelle : « Par logique ensidique, j'entends la logique qui ne connaît comme relations que l'appartenance, l'inclusion, l'implication entre propositions et la logique des prédicats du premier ordre)."( [CAS 93] p.57). Ce qui est ensidique s'oppose à ce qui est " magmatique » (I'humain, l'historique).

18 Atlan précise ([ATL 86], p.63) « ...l'auto-organisation implique nécessairement des interactions entre différents niveaux d'intégration, qui sont en même temps des niveaux différents d'observation. " Avec le co-engendrement des éléments et de la forme, nous avons bien interaction entre deux niveaux différents - et une convergence supplémentaire entre complexité et auto-organisation. 
du recours à des variables au sens scientifique — donc « ensidique » — du terme), c'est le critère du co-engendrement des éléments et de la forme qui est pertinent.

Nous allons maintenant aborder la question à partir d'un autre outillage.

\section{La perspective des boucles autopoiétiques étranges}

Nous allons maintenant envisager la question avec l'apport des boucles autopoiétiques étranges ${ }^{19}$.

\subsection{Boucle cellule (boucle 1)}

Le concept de clôture opérationnelle est introduit par Francisco Varela [VAR 89a] pour décrire la reproduction permanente d'une cellule par elle-même, à partir d'un substrat de réactions chimiques : la cellule est un système autopoiétique — qui se produit, qui se crée lui-même, par opposition aux machines, même compliquées, qui sont allopoiétiques ${ }^{20}$. La membrane cellulaire permet l'établissement de certaines réactions chimiques, qui ont entre autres pour fonction de reconstituer la membrane... (Varela, [VAR 89a] p.21) ${ }^{21}$
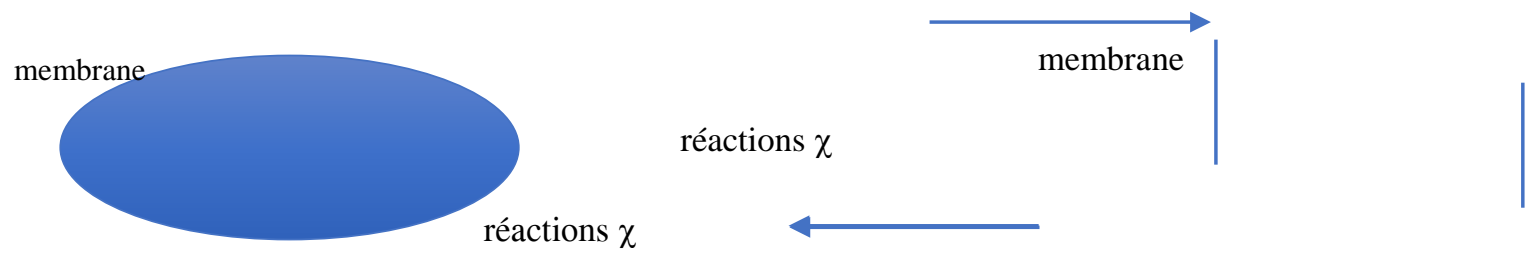

Figure 3. Boucle cellule

Les flèches ont ici la signification d'une production au sens large : la membrane rend possible les réactions chimiques, ces dernières reconstituent la membrane.

Ce fonctionnement décrit une boucle (une causalité circulaire), mais comme la membrane est un englobant, il y a aussi changement de niveau (pas forcément logique) et il faut remarquer que la boucle est étrange ( $c f$. Hofstadter [HOF 85$]^{22}$ ).

La clôture opérationnelle spécifiant une certaine logique interne du système, la prise en compte des échanges avec l'extérieur se perçoit mieux avec l'expression «couplage par clôture opérationnelle ». La notion de couplage structurel, qui correspond à une sélection incessante parmi les structures possibles du système, élargit cette perspective. Le couplage structurel peut se

\footnotetext{
19 Cf. [SAL 20b]

${ }^{20}$ Cette modélisation enrichit la conception de la cellule. Depuis les critères de Piaget [PIA 68], qu'elle vérifie à un point tel qu'on peut penser qu'elle a servi d'exemple pour les concevoir, il est clair que la cellule a les propriétés d'une structure. Puisqu'elle a besoin d'énergie pour survivre, elle vérifie le quatrième critère (énoncé par Lerbet [LER 86] pour passer de la structure au système) et constitue donc un système. La voici, avec Varela, exemple princeps d'une nouvelle catégorie de système.

${ }^{21}$ On voit bien que la clôture opérationnelle n'a pas pour conséquence de clore le système ! La membrane cellulaire ne limite pas un système clos, elle constitue une interface, et permet les échanges avec l'extérieur. Le terme de clôture opérationnelle est à référer aux mathématiques, au sens où l'opération addition, appliquée à l'ensemble des entiers, constitue une telle clôture (opérationnelle au sens propre), puisqu'une telle application ne saurait produire que des entiers.

22 Dans son ouvrage (Gödel, Escher, Bach [HOF 85]), Hofstadter tente de présenter le théorème de Gödel en prenant l'exemple des dessins d'Escher (qui mélangent le niveau du tableau et celui des mains qui le dessinent) et celui des fugues de Bach (au niveau de la mélodie, il y reprise d'un thème, mais au niveau des tonalités, on peut "entendre" un message (comme cette fugue qui présente comme structure de tonalités la succession $\mathrm{B}, \mathrm{A}, \mathrm{C}, \mathrm{H}$ - la tradition anglo-saxonne codant les tonalités, à partir du 'la', à l'aide des majuscules). 
concevoir comme une histoire de la clôture opérationnelle, ou, plus précisément, comme l'évolution au cours du temps de l'interaction système-environnement.

\subsection{Boucles autopoiétiques}

Les systèmes autopoiétiques semblent caractérisés par un fonctionnement en boucle étrange, du type de celle avancée par Varela pour la cellule (cf. supra § 3.1). Si l'on veut être plus précis, Varela ([VAR 89a], p.45) propose cette définition/caractérisation :

«Un système autopoiétique est organisé comme un réseau de processus de production de composants qui (a) régénèrent continuellement par leurs transformations et leurs interactions le réseau qui les a produits, et qui (b) constituent le système en tant qu'unité concrète dans l'espace où il existe, en spécifiant le domaine topologique où il se réalise comme réseau »

Il précise ([VAR 89a], p. 46) : «De même, l'organisation d'un cristal est déterminée par les positions spatiales relatives de ses composants; au contraire, dans une machine autopoiétique, l'organisation est déterminée par les relations, non pas entre les composants, mais entre les processus de production des composants... »

Il donne aussi une définition de la clôture opérationnelle, très proche des énoncés précédents, mettant encore plus l'accent sur les processus ([VAR 89a], p.86) : "Nous dirons d'un système autonome qu'il est opérationnellement clos si son organisation est caractérisée par des processus : dépendant récursivement les uns des autres pour la génération et la réalisation des processus euxmêmes, et constituant le système comme une unité reconnaissable dans l'espace (le domaine) où les processus existent. »

Cette grande ouverture par rapport aux "domaines" et aux processus autorise - et même incite — à utiliser ces outils pour modéliser le fonctionnement sociétal.

\subsection{Boucle 'action/perception'}

Varela, dans les outils qu'il propose, parle de plus en plus du couplage structurel ${ }^{23}$. En 1993 [VAR 93], il met en scène une boucle «action/perception » (p. 235), sans la formaliser (sans faire de schéma). Il parle (au niveau des « schèmes sensori-moteurs récurrents ») de perception, bouclée sur l'action guidée par la perception. Ce qui peut se schématiser ainsi :

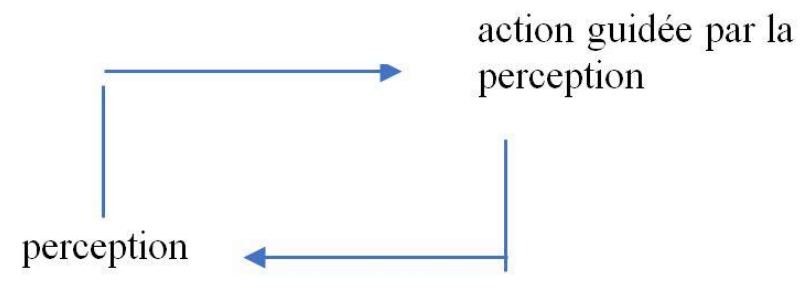

Figure 4. Boucle action/perception

Les flèches ont ici la signification d'une production au sens large : la perception guide l'action, cette dernière intervient en régulant la perception.

Pour être plus près de la pensée de l'auteur, il faut compléter ce schéma sur les schèmes sensorimoteurs (bouclés) par les structures cognitives, en aval ${ }^{24}$. Par rapport à la boucle étrange qui

\footnotetext{
${ }^{23}$ Qualifié de 'structural' dans l'ouvrage de 1994 [MAT 94] p.65, au lieu de 'structurel', mais il s'agit du même concept.

24 «En bref, cette approche (l'enaction) se compose de deux points : 1) la perception consiste en une action guidée par la perception; 2) les structures cognitives émergent des schèmes sensori-moteurs récurrents qui permettent à l'action d'être guidée par la perception. " 
concernait la cellule, “l'englobement" n'est pas physique, n'est pas lié à l'espace mais au fonctionnement : c'est la perception qui est omniprésente, même si elle évolue — c'est ainsi que le couplage structurel fonctionne. Par analogie avec la boucle "cellule" (les réactions chimiques reconstituent en permanence la membrane cellulaire, cette dernière englobant la cellule et permettant par-là aux réactions chimiques de fonctionner), on pourrait dire que la perception englobe l'action.

Cette centration sur les boucles permet d'esquisser un rapprochement entre Varela et Edelman. Ce dernier [EDE 92] élabore une théorie qu'il nomme la TSGN (théorie de la sélection des groupes neuronaux) ${ }^{25}$. Dans la complexité de notre système nerveux, il propose de discerner les groupes neuronaux et les cartes neuronales. Les premiers sont fondés sur une propagation de l'excitation due à la proximité (les neurones voisins d'un neurone excité ont des chances de l'être aussi), mais construisent une dynamique propre : «Nous pouvons donc résumer la situation en disant que, en général, aucun neurone n'est sélectionné isolément dans une carte, aucun neurone n'est connecté de façon réentrante à un unique neurone d'une autre carte ; qu'aucun neurone isolé ne présente les propriétés qu'il présente au sein d'un groupe. » ([EDE 92] p.136) Les secondes correspondent à une fonctionnalité (l'accomplissement d'un geste, par exemple). Les cartes interagissent grâce à un processus appelé réentrée, qui amène l'émergence de propriétés nouvelles. Lorsqu'un sujet agit, il met ainsi en jeu à la fois des groupes neuronaux et des cartes neuronales, cet ensemble étant nommé cartographie globale. Une cartographie globale est une structure dynamique qui couple les sorties d'une multitude de cartes interconnectées de façon réentrante au comportement sensori-moteur.

Ainsi, avec la propriété de réentrée nous retrouvons ce qui caractérise un fonctionnement en boucle, et en boucle avec changements de niveaux, ce qui fait de ces boucles des boucles étranges. Cette convergence avec Varela est d'autant plus nette que l'interconnexion réentrante au comportement sensori-moteur ressemble fort à la boucle 'action/perception'. Varela [VAR 93] référencie d'ailleurs Edelman. Changeux ([CHA 83]) insiste lui aussi sur l'importance des boucles ré-entrantes.

\subsection{Fonctionnement d'une société, boucle 3}

\subsubsection{Interaction}

Ce qui se joue lors de la rencontre de deux êtres humains s'appelle une interaction. En proposant de définir la représentation comme ce qui permet de véhiculer une telle interaction ${ }^{26}$, on peut penser à s'appuyer sur la notion d'interaction en Physique ${ }^{27}$. D'autant plus si on constate qu'en sciences humaines et sociétales, la notion «d'interaction sociale » est couramment évoquée mais non définie. Pour la caractériser en tenant compte des ouvrages de Physique, on constate que l'action de contact ne pose pas problème, mais que l'action à distance en pose, et que la notion d'interaction (liée à une théorie du champ) a été élaborée pour cette situation (d'action à distance). On retrouve cette nuance en sciences humaines et sociétales. Lorsqu'il est question de coups ou de caresses, concevoir l'interaction (de contact) ne pose guère de problème. Mais en l'absence de contact, concevoir que l'interaction s'établit par échange de représentations ouvre une fenêtre pour la modélisation ${ }^{28}$.

\footnotetext{
25 « Si nous admettons que les fonctions cérébrales sont bâties selon un processus sélectif, nous devons alors être capables de réconcilier la variabilité structurelle et fonctionnelle du cerveau avec la nécessité d'expliquer comment il effectue des catégorisations. » ([EDE 92] p.127); voir aussi [SAL 20a].

26

cf. [SAL 96], [SAL 15].

27 Précisons ce qu'est, en Physique, la grandeur action. Un homme pousse un objet et le déplace. Il a exercé sur lui une force $F$, et plus précisément une force durant un intervalle de temps ?t et sur une distance $d, c^{\prime}$ est-à-dire une action - le produit $F$. $d$ est un travail W (ou une énergie), le produit W. ?t est une action (la grandeur action, en Physique, a la dimension 'énergie que multiplie temps' - l'unité de travail étant le joule (J), celle de temps étant la seconde (s), l'unité d'action est le joule-seconde (J.s).

${ }^{28}$ En renvoyant à [SAL 18b], chap II, pour une revue de questions à la hauteur du problème, signalons les références les plus marquantes : Bruhat ([BRU 67]), Landau \& Lifchitz, ([LAN 66]), Lévy-Leblond \& Balibar ([LEV 84]) pour l'interaction en Physique ; (C) 2021 ISTE OpenScience - Published by ISTE Ltd. London, UK - openscience.fr 
Si l'on fait le bilan du concept de la Physique, on peut repérer plusieurs caractéristiques de l'interaction :

La réciprocité.

Le principe de l'égalité (de l'action et de la réaction) qui, s'il peut sembler de prime abord inadapté aux échanges entre humains, mérite d'être médité.

Le lien (ou même l'identité) entre champ et interaction.

Le caractère incontournable : il s'agit d'action (ou de force), ce qui produit un effet.

En s'appuyant sur le dernier caractère on peut affirmer que l'interaction n'a rien à voir avec la communication — ou tout au moins avec le caractère euphémisé qu'on lui donne trop souvent : un responsable politique qui annonce des mesures (des décisions) qui sont mal reçues aurait commis une erreur de «com», ou aurait «mal géré sa com»... L'interaction entre deux sujets humains peut être de contact (gifle ou bien pire !), et remarquons qu'une représentation peut faire autant de mal, sinon plus, qu'un coup de poing ou de pied — même lorsqu'elle n'est pas énoncée ! Dans le cas de figure de la représentation, le temps de propagation peut être aussi bref que celui d'un coup de poing, mais il peut être beaucoup plus long (le temps qu'elle soit comprise, dans tous ses attendus et prolongements éventuels). Un concept (ou une notion) d'interaction en sciences humaines et sociétales ne peut être pertinent qu'à la condition d'inclure la possibilité d'action de contact (physique, concrète), ainsi que celle d'action à distance (symbolique, utilisant des représensations). On retrouve de fait la même difficulté qu'en Physique avec l'interaction à distance (sans contact). Et là encore, on peut considérer le contact comme un cas limite de l'interaction à distance.

En résumé, un échange à intensité faible ((à investissement énergétique faible) constitue une rencontre mineure, à risque mineur. Une rencontre à intensité forte (à investissement énergétique fort) constitue une rencontre majeure, le risque est lui aussi majeur : l'être humain peut en « sortir » abimé ou grandi. Ces effets possibles renvoient à ce qui a été nommé plus haut le "caractère incontournable, qui est celui de la confrontation à des forces.

Le caractère de réciprocité est bien présent lui aussi. Dans une interaction (dans une rencontre), même celui qui est immobile et se tait envoie des messages. Bien entendu, le principe d'égalité de l'action et de la réaction, qui, appliqué à la rencontre entre deux humains, semble vérifié dans les cas où les deux sont dans une dynamique symétrique (il rappelle aussi fortement les règles de l'échange soulignées par l'anthropologie), ne semble pas pouvoir être généralisé. On trouve des interactions à dynamique 'dominant-dominé', où en tout cas l'un semble agir, parler, décider plus que l'autre. Il faut d'abord se méfier des apparences. Ce n'est pas forcément celui qui parle tout le temps, par exemple, qui est dans une position dominante ou plus assurée. Cette profusion de paroles, ces interventions réitérées peuvent être l'indice d'une position qui cherche à s'affermir. Mais en admettant que l'on peut discerner le «profond» sous la «surface» et qu'il semble bien y avoir déséquilibre entre les forces exercées, maintenons qu'il est judicieux de méditer sur le principe précédent.

Enfin, le lien entre champ et interaction exige, pour être exploré, la prise en compte de la culture.

\subsubsection{Double mouvement}

Les interactions entre humains, au sein d'une société, sont incessantes et quasiment innombrables. On peut parler à ce propos d'interactions distribuées, en se fondant sur la notion de distribution pour les neurones, (une propriété émergente, telle que la représentation de quelque chose, ou telle qu'une idée, est distribuée sur un grand nombre de neurones). Le deuxième moment 
du schéma dialectique en trois moments, celui de la Particularité, est fondé sur cette distribution des interactions. Mais de la même façon que chaque interprétation d'une règle est structurée par la règle elle-même, chaque interaction est structurée par la culture (généralisation à la langue et à la culture du fonctionnement en trois moments).

D'où ce que l'on peut appeler le double mouvement. Toute modélisation du fonctionnement de la société, pour être pertinente, doit tenir compte de ce double mouvement qui caractérise le fonctionnement de la culture : d'un côté, la culture est sans cesse reconstruite par les interactions entre sujets humains (autopoièse sociétale, et/ou passage au troisième moment, celui de la Singularité); mais de l'autre, ces interactions sont structurées par la culture, que chaque sujet humain a « en lui ».

\subsubsection{Boucle 'distribution des interactions/culture' (boucle 3)}

Le fonctionnement que nous avons désigné par double mouvement peut se schématiser par une boucle analogue aux boucles autopoiétiques des $\S 3.1$ et 3.3 (boucle cellule (Fig. 3) et boucle 'action/perception'(Fig. 4) :

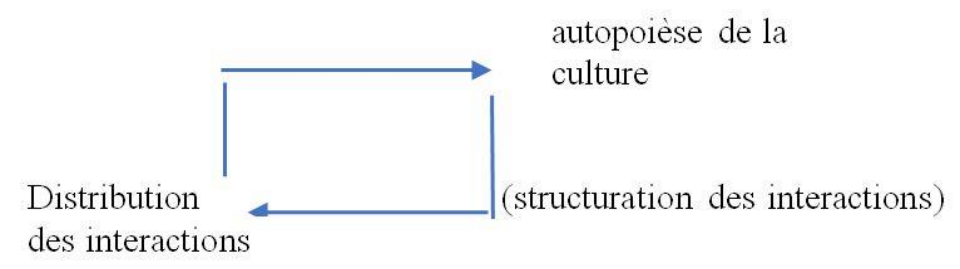

Figure 5. Boucle 'distribution des interactions/culture

Les flèches ont ici la signification d'une production au sens large : la distribution des interactions produit l'autopoièse de la culture, cette dernière structure toute interaction.

Ici, c'est la culture qui est omniprésente même si elle se reproduit tout en évoluant, puisqu'elle structure les interactions — on retrouve l'idée d'englobement, même si ce dernier a des spécifications quelque peu différentes d'une boucle à l'autre. Cela dit, dans le cas de la boucle 'action/perception', on pourrait dire aussi que la perception structure l'action.

\subsection{Boucle 3 et schéma II}

La boucle 2 ('action/perception', § 3.3, Figure 4) fonctionne au niveau logique individuel, la boucle 3 (boucle 'distribution des interactions/culture', Figure 5) au niveau logique collectif, car si une interaction entre deux humains peut être considérée comme se trouvant au niveau logique individuel (ou au niveau logique de l'élément interaction), la distribution des interactions place clairement le fonctionnement de cette troisième boucle au niveau logique collectif (ou au niveau de l'ensemble des interactions).

Si l'on veut être plus précis en examinant plus avant la complémentarité de l'outil boucle 'perception/action' avec la description par clôture opérationnelle et couplage structurel, il faut tout d'abord reprendre le 'b' de la caractérisation du système autonome opérationnellement clos : le système doit être «repérable » en tant qu' " unité reconnaissable dans le domaine où les processus existent ». Le système ici est le système nerveux d'un sujet humain, ou le sujet humain lui-même.

Pour préciser également le repérage du système correspondant à la boucle 'distribution des interactions/culture', il faut dire qu'il s'agit d'une société, munie de la culture qui lui est propre (qui la caractérise). 
Ainsi, ces deux boucles modélisent, de manière analogue, pour l'une un fonctionnement situé au niveau logique individuel et pour l'autre un fonctionnement qui concerne (et qui caractérise) le niveau logique collectif.

Cela dit, la boucle 3 modélise le même fonctionnement que les schémas I et II (schéma en trois moments, schéma en trois moments appliqué à la langue, aux institutions et à la culture). Or, avec l'interprétation du $3^{\mathrm{e}}$ moment rappelée au $\S 2-3$ (dès l'émergence d'une règle nouvelle, d'une nouvelle façon de parler, de penser, cette nouvelle forme entre dans l'interaction entre déjà-institué et capacité instituante), le schéma en trois moments fonctionne en boucle ${ }^{29}$. On pourrait faire remarquer que si le schéma en trois moments a une structure ternaire, la boucle 3 fait apparaître, à première vue, deux "nœuds" seulement dans la boucle. Mais on pourrait insérer dans la boucle un troisième nœud (structuration des interactions) au lieu d'indiquer cette dynamique sur la flèche.

On peut poursuivre un peu l'homologie entre les deux outils de modélisation. La distribution des interactions correspond bien sûr au moment du Particulier, l'autopoièse de la culture à celui du Singulier. Et la structuration des interactions, c'est bien sûr le rôle du déjà institué et du moment de l'Universel.

\section{Possibilité de définition}

Nous avons exploré l'approche de la complexité à partir du co-engendrement des éléments et de la forme - qui s'appuie sur la théorie de l'institution et le schéma en trois moments. Puis nous avons repris cette approche avec les boucles autopoiétiques étranges, en montrant que les deux pistes convergeaient. Le pas suivant consiste à tenter un énoncé de définition (de la complexité ou d'un système complexe).

\subsection{Reprise des deux pistes}

Si nous reprenons la suggestion de Castoriadis [CAS 93], à savoir la caractérisation de la complexité par le co-engendrement des éléments et de la forme, nous avons le choix entre deux possibilités : ou bien réserver le label complexité au strict processus de co-engendrement, à son advenue, ou bien parler de système complexe dès lors que la structure du système rend ce coengendrement possible.

Dans le choix deux, un système complexe serait caractérisé (voire défini) par un fonctionnement décrit par le schéma en trois moments de type II, ou par la boucle autopoiétique étrange 3.

Et la boucle 2, peut-on la questionner dès lors ? Car si elle a même structure que la boucle 3 , peut-on repérer un co-engendrement des éléments et de la forme dans cette boucle, autrement dit dans l'humain ? Castoriadis [CAS 93] nous y encourage, puisque l'un de ses deux exemples ( $c f$.

\footnotetext{
${ }^{29}$ Pour être plus précis, on peut rappeler les compléments à apporter au schéma en trois moments pour s'assurer de sa capacité à modéliser un fonctionnement autopoiétique (cf. [SAL 18b], p.178) :

"La rencontre des deux paradigmes que sont la théorie des systèmes et la théorie de l'institution - rencontre évoquée et décrite par Sallaberry ([SAL 99]) - peut être considérée comme inéluctable, puisque les deux théories modélisent des situations à interactions multiples - même si, au départ, les situations évoquées (ou choisies) par chacun des deux théories étaient (ou semblaient) éloignées. Le travail de réflexion sur cette rencontre a été largement amorcé par Barel [BAR 75] et Castoriadis [CAS 93]. À leur suite, nous reprenons ce travail et nous le poussons un peu (un pas) plus loin, en montrant que le schéma en trois moments, qui modélise le fonctionnement d'une forme, avec les précisions (ou compléments) apportés :

o sur la conception dynamique du troisième moment

o sur l'effet Mülhmann

o sur la réciprocité institutionnelle

o sur le caractère dynamique et grave des interactions

o sur la distribution des interactions qui reconstruit en permanence la culture

o sur la structuration de toute interaction par la culture,
} 
supra § 2.2.4) est le " «cercle» ADN-protéines", ses deux exemples (ce «cercle» et la polis) englobant donc non seulement l'humain et le sociétal, mais allant plus loin dans le domaine du vivant. La boucle 2 ('action /perception'), travaillant bien sur deux niveaux ${ }^{30}$, comme toute boucle étrange, crée bien des formes à partir d'éléments plus frustes - restructure ces éléments, donc, en leur conférant un sens ${ }^{31}$. Comme déjà dit, elle rappelle et s'appuie sur les boucles ré-entrantes évoquées par Changeux [CHA 83], Edelman [EDE 92], Varela [VAR 93]. En reportant à plus tard un examen plus systématique des différents types de systèmes de ce point de vue (structure en boucle étrange et possibilité ou non d'un co-engendrement...), nous avons un argument pour affecter à l'humain, comme au sociétal, le caractère de complexe - sur la base du critère du coengendrement des éléments et de la forme.

L'important, c'est que l'état global (d'une règle, d'une institution, d'un système...) dépend des états locaux, qui dépendent eux-mêmes de l'état global. Castoriadis ([CAS 93]) insiste sur cette réciprocité et sur cette restructuration des états locaux par l'état global: «La combinaison d'éléments d'un niveau donné pourrait, sous certaines conditions, faire apparaître un niveau émergent - et l'on ne semble pas s'inquiéter outre mesure de la question des interactions entre ce niveau émergent et les niveau «antérieurs » (ou «inférieurs »)» (p. 57) ; et, plus loin (p. 58) «Il devrait donc y avoir action unique du «bas » vers le « haut», et jamais du « haut » vers le «bas »... Mais, évidemment, il y a action du «haut» vers le «bas »: vous m'injuriez et je vous gifle, une idée me vient et je l'écris. » Nous sommes bien dans l'idée que la forme qui émerge restructure l'ensemble du système — c'est en ce sens que l'on peut parler de forme ${ }^{32}$.

Il faut aussi rappeler qu'une forme est toujours - fût-ce en partie - imaginaire (hommage, à nouveau, à Castoriadis [CAS 75]). De façon plus prosaïque, c'est dire que toute forme existe en tant que représentation - même si elle a des aspects réels, concrets. Le Panthéon, par exemple, mais aussi la Sorbonne, sont repérables en tant que bâtiments, mais convoient une évocation lourde de symboles - c'est leur aspect imaginaire, leur aspect représentation. Ce qui veut dire que la création d'une forme nouvelle se fonde sur l'imaginaire.

Le co-engendrement fonctionne à la réciprocité institutionnelle. Les conventionnels instituent la République - ils sont portés par l'ample mouvement qui a soulevé Paris mais aussi la France. Mais simultanément, chacun, qu'il ait été ci-devant marquis ou ci-devant sans-culotte, devient citoyen! Les chacunes deviennent, elles aussi, citoyennes, mais il leur faudra un siècle et demi pour récupérer le droit de vote...

\subsection{En résumé}

Dans le choix 2 (parler de système complexe dès lors que la structure du système rend le coengendrement des éléments et de la forme possible), on peut donc proposer de définir un système complexe comme un système qui fonctionne en boucle autopoiétique étrange - comme un système susceptible de déclencher un co-engendrement éléments -forme.

\footnotetext{
${ }^{30}$ (« 1) la perception consiste en une action guidée par la perception ; 2) les structures cognitives émergent des schèmes sensorimoteurs récurrents qui permettent à l'action d'être guidée par la perception. » Varela , [VAR 93], p.235.

${ }^{31}$ Sans vouloir s'engager trop dans la question des rapports entre représentation et perception, reprenons la position de Bruner [BRU 58]. En résumé, la perception est conçue comme 'représentation plus catégorisation'. Prenons l'exemple d'une circulation en voiture, de nuit. Le conducteur pilote en surveillant la route, et notamment le bas-côté. A priori, c'est continu : herbe assez rase, quelque chose de plus sombre qui indique un fossé, adossé à un talus... Brusquement, une discontinuité apparaît : une tache plus sombre sur le bas-côté. L'attention se concentrant, il perçoit deux points brillants sur la tache sombre. II les identifie aussitôt comme les yeux d'un animal, ce qui lui permet de restructurer l'ensemble des éléments pour créer une forme : il s'agit d'un animal! Tel détail qui restait bizarre et flou peut désormais prendre le sens d'une queue, ou de la tête... La restructuration crée une forme, qui donne sens aux éléments.

32 Pour plus de précisions sur cette notion de forme, cf. [SAL 10]. 
Première formulation: Un système complexe est un système qui fonctionne en boucle autopoiétique étrange.

En pensant à l'encéphale humain et aux précisions de Changeux [CHA 83], Edelman [EDE 92], Varela [VAR 93], on pourrait aussi dire qu'un système complexe est structuré sur une boucle autopoiétique étrange, ou sur l'articulation de plusieurs boucles autopoiétiques étranges. La figure à plusieurs boucles est aussi pertinente dans le cas d'un système humain-sociétal, puisqu'aux boucles intra-encéphale s'articule la boucle 3 .

Seconde formulation: un système complexe est un système structuré sur une boucle autopoiétique étrange, ou sur l'articulation de plusieurs boucles autopoiétiques étranges.

\section{Discussion : la question de l'invariant}

Pour engager une discussion sur ce qui précède, poser la question de l'invariant semble non seulement pertinent, mais encore incisif voire provocateur.

\subsection{Vergnaud}

Vergnaud ([VER 85] et [VER 91]) considère que tout concept est caractérisé par une structure $(\mathrm{S}, \mathrm{I}, \mathrm{S})$ :

- S renvoie aux situations pour lesquelles le concept est pertinent.

- I désigne l'invariant qui justifie la construction du concept (les exemples de l'énergie, de la quantité de mouvement, suffisent à convaincre que souvent c'est le repérage d'un invariant qui déclenche l'élaboration d'un concept).

- S rappelle que tout concept est désigné par un mot, voire par une abréviation (symbolisation).

\subsection{Invariant et concepts de la physique}

Cette idée de lier un concept à un invariant (ou à un groupe d'invariants) éclaire les concepts des sciences physiques avec une grande pertinence, en montrant, pour chacun, sa nécessité dans la situation sur laquelle il travaille. Ainsi, on peut dire que c'est parce que beaucoup d'actions ou de transformations s'effectuent à quantité de matière constante (l'invariant est la quantité de matière) que le concept de masse a été inventé : sa fonction est d'exprimer l'invariance de la quantité de matière $^{33}$. De même, la chaleur et le travail mécanique s'échangeant avec conservation de la quantité dans toutes les transformations connues, le concept d'énergie désigne ce qui change de forme (passant de l'électrique au chimique, ou au mécanique, ou encore au thermique, et vice versa) tout en se conservant. Lorsqu'un circuit électrique n'est pas très long (tous les circuits électriques, exceptées les lignes de transport), le débit des charges électriques est le même en chaque point du circuit, à chaque instant. Le concept d'intensité du courant électrique est chargé d'exprimer cette invariance.

\subsection{La structure et le système}

L'énoncé, par Piaget, des trois critères qui caractérisent une structure (totalité, transformation, autoréglage) amène la notion de structure au bord du concept (il suffirait d'un énoncé strict de définition, du genre "on appelle structure tout "objet" caractérisé par les trois critères...").

\footnotetext{
33 Bien entendu, on sait depuis un demi-siècle environ que les réactions chimiques ne s'effectuent pas rigoureusement en conservant la quantité de matière - contrairement à ce qui avait été énoncé par Lavoisier fin XVIIle, à partir de nombreuses mesures (les siennes, celles de Priestley, d'autres...). Mais la quantité de matière qui ne se conserve pas n'est pas à la portée de nos moyens de mesure. L'approximation qui consiste à poser l'invariance de la quantité de matière est ainsi pertinente pour l'ensemble des réactions chimiques. 
Lerbet [LER 86] propose de caractériser le système grâce aux trois critères, plus celui d'énergie (un système ne fonctionne pas sans énergie). Le Moigne [LEM 90] a une approche plus imagée ; pour lui, un système est « quelque chose qui, dans un environnement actif, semble poursuivre un but » (exemple d'environnement actif : région polaire; exemple de but: survivre ; exemple de système : l'ours blanc). Les deux approches, bien qu'elles partent de points différents, sont compatibles et convergent.

Mais ce qui nous intéresse maintenant est l'importance du troisième critère, celui d'autoréglage. En effet, il introduit un invariant d'un type nouveau, un invariant lié au fonctionnement. Un tel invariant, caractérisant structure et système, se révèle être spécifique du vivant, donc de l'humain et, partant, du sociétal. Il ouvre la possibilité même de la biologie moderne, comme des sciences humaines et sociétales ${ }^{34}$.

\subsection{Le système complexe}

Tout système est donc caractérisé par un invariant du type fonctionnement, l'autoréglage, mais cela ne suffit pas à le caractériser en tant que système complexe. La question qui se pose est, dès lors, celle de la possibilité d'un invariant spécifique aux systèmes complexes.

Si nous reprenons ce qui précède, à savoir la suggestion de Castoriadis [CAS 93] (liée au coengendrement des éléments et de la forme) et le constat (ou l'hypothèse si l'on veut être prudent) qu'un système :

dont le fonctionnement est décrit par le schéma en trois moments (schéma II),

structuré en boucles autopoiétiques étranges (du type boucle 2 ou du type boucle 3 ),

rend possible ce co-engendrement, on peut proposer que l'invariant qui caractérise un système complexe est ce type de boucle — ou si l'on préfère d'être organisé sur ce type de boucle.

Nous retrouvons alors avec plaisir les trois 'principes/critères' de Morin [MOR 90] — ce qui est rassurant :

le critère (le principe) de récursivité (grâce à l'aspect boucle) ;

le critère hologrammatique (la forme organise les éléments, qui font émerger la forme) — le critère hologrammatique peut ainsi être interprété comme exprimant la réciprocité institutionnelle ;

quant au principe dialogique, nous sommes bien, avec ces outils de modélisation (et avec ces trois principes/critères), en train de traverser (justement) ${ }^{35}$ les trois principes de la logique formelle (identité, non-contradiction, tiers exclus).

D'aucuns trouveront peut-être que ce genre d'invariant est le contraire des invariants « habituels », ou classiques. On peut d'ailleurs penser cela dès l'invariant de la structure et du système - invariant lié au fonctionnement, donc invariant éminemment dynamique... À plus forte raison, il n'est pas surprenant que l'invariant correspondant à la complexité soit quelque peu étonnant... La complexité ne serait pas si l'on pouvait l'affubler d'un invariant classique.

\footnotetext{
34 cf. [SAL 18b].

${ }^{35}$ Le préfixe grec ?]? (dia) signifie 'à travers'. 'Dialogique' signifie donc (fût-ce partiellement, le sens étymologique ne faisant pas nécessairement force de loi, mais étant difficile à éliminer...) 'à travers la logique' ou 'à travers le logos'... 


\section{Conclusion}

Après un début consacré à une caractérisation de la complexité, prenant appui sur Le Moigne et Morin [LEM 99], deux propositions de définition (celle de Nicolis-Prigogine [NIC 92] et celle de Castioriadis [CAS 93]) ont été exposées, ainsi qu'une nouvelle approche, à partir des boucles autopoiétiques ([VAR 89a] et [VAR 93]). La convergence constatée entre cette approche et celle de Castoriadis a permis l'esquisse d'une définition (pour un système complexe).

La discussion s'est engagée à partir de la question du lien invariant-concept ( $c f$. la proposition de Vergnaud [VER 85] et sa pertinence pour les concepts de la Physique). Le critère d'autoréglage l'une des caractéristiques de la structure et du système - était dèjà repéré comme invariant pour ces deux "objets". Pour un système qualifié de complexe, l'invariant qui découle de la définition proposée est la présence d'une boucle autopoiétique étrange, qui structure un tel système. La conséquence étant la congruence avec les trois critères de Morin [MOR 90] (principe de récursivité, critère hologrammatique, principe dialogique), ce type d'invariant comme la proposition de définition semblent pertinents.

\section{Références bibliographiques}

[ATL 79] Atlan H., Entre le cristal et la fumée, Paris, Seuil,1979.

[ATL 86] Atlan H., A tort et à raison, Seuil, 1986.

[ATL 11] Atlan H., Le vivant post-génomique — ou qu'est-ce que l'auto-organisation ? Paris, Odile Jacob, 2011.

[BAG 17] Bagur T. \& Portocalli G., L'individu et l'interaction, entre rôle social et identité, in Revue européenne de Coaching, $\mathrm{n}^{\circ} 2$, Avril 2017.

[BAR 75] Barel Y., La ville médiévale — système social, système urbain, Grenoble, PUG, 1975.

[BAR 87] Barel Y., La quête du sens. Paris : Seuil, 1987.

[BAR 89] Barel Y., Le paradoxe et le système. Grenoble : PUG. (re-éd.), 1989.

[BAR 93] Barel Y., Système et paradoxe (Autour de la pensée d'Yves Barel). Paris : Seuil, 1993.

[BAC 34] Bachelard G., Le nouvel esprit scientifique, Paris, PUF, 1934

[BAC 38] Bachelard G., La formation de l'esprit scientifique, Parais, Vrin, 1938.

[BRU 62] Bruhat, G., Cours de Physique générale - Thermodynamique (remaniée par Kastler A. et Vichnievsky R.), Paris, Masson, 1962.

[BRU 67] Bruhat G., Cours de Physique générale - Mécanique (revue et complétée par Foch A.), Paris, Masson, 1967.

[BRU 58] Bruner JS., Les processus de préparation à la perception, in Études d'épistémologie génétique s/d Piaget, J. tome VI Logique et Perception, Paris, PUF, 1958.

[CAR 65] Cardan P., Marxisme et théorie révolutionnaire, in Socialisme ou Barbarie, n³9 (mars-avril) et 40 (juin-aôut), 1965.

[CAS 75] Castoriadis C., L’institution imaginaire de la société. Paris : Seuil, 1975.

[CAS 78] Castoriadis C., Les carrefours du labyrinthe. Paris : Seuil,1978.

[CAS 93] Castoriadis C., Complexité, magmas, histoire. In Système et paradoxe. Paris : Seuil, 1993.

[CHA 83] Changeux JP., L'homme neuronal, Paris, Fayard, 1983.

[CHA 02] Changeux JP., L'homme de vérité, Paris, Odile Jacob, 2002.

[EDE 92] Edelman G.M. Biologie de la conscience, tr.fr. Paris, Odile Jacob, 1992.

[ESP 84] Espagnat B. d', La non-séparabilité ou l'insaisissable réalité, Le monde quantique, s/d Deligeorges, Paris, Seuil, p.141$146,1984$.

[FUR 78] Furet F., Penser la Révolution française, Paris, Gallimard, 1978.

[GOF 73] Goffmann E., La mise en scène de la vie quotidienne, Paris, Minuit, 1973. 
[GOF 93] Goffman E., La communication en défaut, Actes de la recherche en sciences sociales, Volume 100, $\mathrm{n}^{\circ} 1, \mathrm{p} / 66-72$, 1993.

[HES 81] Hess R. \& Savoye A., L'analyse institutionnelle, Paris, PUF (Que sais-je ?), 1981.

[HOF 85] Hofstadter D., Godël-Escher-Bach, Paris, Interéditions, 1985.

[LAN 66] Landau L. \& Lifchitz E., Théorie du champ, Moscou, Éd. MIR, 1966.

[LEM 90] Le Moigne JL., La modélisation des systèmes complexes, Paris, Dunod, 1990.

[LEM 95] Le Moigne JL., Les épistémologies constructivistes, Paris, PUF (Que sais-je?), 1995.

[LEM 99] Le Moigne JL. \& Morin E., L intelligence de la complexité, Paris l’Harmattan (Cognition et Formation), 1999.

[LER 86] Lerbet,G., De la structure au système, Maurécourt : Ed. Universitaires, 1986.

[LEV 84] Levy-Leblond JM \& Balibar F., Quantique, Paris, Interéditions et CNRS, 1984, - réed Masson, 1997.

[LEV 58] Levi-Strauss C., 1958, Anthropologie Structurale, Paris, Plon, 1974.

[LOU 70] Lourau R., L'Analyse institutionnelle, Paris, Ed. de Minuit, 1970.

[MAR 89] Marc E. \& Picard D., L interaction sociale, Paris, PUF, 1989.

[MAT94] Maturana HR. \&Varela FJ., L'Arbre de la connaissance, Ed. Addison-Wesley France, 1994

[MEA 34] Mead GH., 1934, L'Esprit, le soi et la société, Paris PUF, 1963.

[MOR 90] Morin E., Introduction à la pensée complexe, Paris, ESF, 1990.

[MÜL 61] Mülhmann W., Messianismes révolutionnaires du Tiers Monde, 1961, tr.fr. Gallimard, 1968.

[NIC 92] Nicolis G. \& Prigogine I., A la rencontre du complexe, Paris, PUF., 1992.

[NIZ 05] Nizet J. \& Rigaux N., La sociologie de Erving Goffman, Paris, La Découverte, 2005.

[PIA 68] Piaget J., Le structuralisme, Paris, PUF, 1968.

[PRI 79] Prigogine I. \& Stengers I., La nouvelle alliance : métamorphose de la science, Paris, Gallimard, 1979.

[PRO 28] Propp V., Morphologie du conte, 1928, tr. fr. Paris, Seuil, 1965.

[SAL 96] Sallaberry JC, Dynamique des représentations dans la formation, Paris, L’Harmattan (Cognition et Formation).,1996.

[SAL 99] Sallaberry JC, “Théorie des systèmes et théorie de l'institution : la rencontre de deux paradigmes ", L’année de la recherche en sciences de l'éducation $n^{\circ} 1999$, p.5-25, 1999.

[SAL 03] Sallaberry JC,. Théorie de l'institution et articulation individuel-collectif, in Actualité de la théorie de l'institution hommage à René Lourau s/d Ardoino, J., Boumard, P., Sallaberry, JC., Paris, L'Harmattan (Cognition et Formation), p.75-110, 2003.

[SAL 10] Sallaberry JC., L'émergence des formes dans les groupes, in L'émergence des formes d'organisation dans les groupes de formation, s/d Sallaberry JC \& Vannereau J., Paris, L’Harmattan (Cognition et Formation). 2010.

[SAL 15] Sallaberry, JC,. La représentation : une synthèse (un concept) possible ? - Note de synthèse, L 'Année de la recherche en sciences de l'éducation, $n^{\circ} 2015, \mathrm{p} .213-256,2015$.

[SAL 18a] Sallaberry,JC., TSGN (Edelman) et apprentissage de la lecture, in Ingénierie cognitique Volume 18 - 2 Numéro 1 , https://www.openscience.fr/Ingenierie-cognitique, 2018a.

[SAL 20a] Sallaberry JC., Cognition individuelle et cognition collective, in Ingénierie cognitique, volume 20- 4 Numéro 1, https://www.openscience.fr/Ingenierie-cognitique, 2020a.

[SAL 20b] Sallaberry JC., Boucles autopoiétiques étranges, in L'Année de la recherche en sciences de l'éducation, $n^{\circ} 2020$ (Éducation et cognition — des éléments théoriques amenés par les diverses modélisations à la pédagogie Freinet), p.203-212, 2020b.

[SAL 20c] Sallaberry JC., Modélisation et pertinence, in L 'Année de la recherche en sciences de l'éducation, $n^{\circ} 2020$ (Éducation et cognition — des éléments théoriques amenés par les diverses modélisations à la pédagogie Freinet), p.55-58, 2020c.

[SAL 18b] Sallaberry JC. \& Claverie B., Introduction aux sciences humaines et sociétales, Paris, L'Harmattan (Cognition et Formation), 2018.

[SAU 15] Saussure F., Cours de linguistique générale, 1915, tr.fr. Paris, Payot, 1972.

[VAR 89a] Varela F.J., Autonomie et connaissance, Paris, Seuil, 1989a. 
[VAR 89b] Varela F.J., Connaître les sciences cognitives, Paris, Seuil, 1989 b.

[VAR 93] Varela F.J., Thompson E. \& Rosch E., L'inscription corporelle de l'esprit, Paris, Seuil, 1993.

[VAR 95] Varela F.J., Connaissances et représentations, in Dossier : Les Sciences de l'homme, B.I.C. (Bulletin d'Information des Cadres, EDF-GDF) n 27, mars 1995, p.78-88, 1995.

[VER 85] Vergnaud G., Concepts et schèmes dans une théorie opératoire de la représentation, in Psychologie Française, $\mathrm{n}^{\circ}$.spécial "Les représentations, 1985.

[VER 91] Vergnaud,G., La théorie des champs conceptuels, in Recherches en didactique des mathématiques, 10, p.133-170, 1991.

[VID 92] Vidal C., La réaction chimique créatrice de rythmes et de formes, BUP n 742 (mars 1992), p. 341-362, 1992.

[WHI 10] Whitehead A.N. \& Russel B., Principia Mathematica, Cambrige, Cambridge Mathematical Library, 1910. 